\title{
Propuesta para la valoración de pymes en Colombia. Estudio de caso en el sector comercial e industrial ${ }^{1}$
}

\author{
Miguel Ángel Laverde Sarmiento ${ }^{2}$ \\ Juan Carlos Lezama Palomino ${ }^{3}$ \\ Jorge Fernando García Carrillo ${ }^{4}$
}

Recibido: 3 de julio del 2018

Aprobado: 30 de agosto del 2018

\section{Clasificación JEL: G12, D46, M40}

\footnotetext{
1 Laverde, M. A., Lezama J. C., y García J. F. (2019). Propuesta para la valoración de pymes en Colombia. Un estudio de caso en el sector comercial e industrial. Revista Activos, 16(31), 19-54. DOI: https://doi.org/10.15332/25005278.5315
}

2 Contador público, docente de la Facultad de Contaduría Pública de la Universidad Santo Tomás, Bogotá D. C., Colombia. Miembro del grupo de investigación: Contaduría: información, control e impacto social. Correo electrónico: miguellaverde@usantotomas.edu.co - ORCID: https://orcid.org/0000-0002-60069195

3 Administrador de empresas, contador público y candidato a grado de la Maestría de Ciencias Económicas, Universidad Nacional de Colombia. Docente e investigador de la Facultad de Contaduría Pública, Universidad Santo Tomás, Bogotá D. C., Colombia. Miembro del grupo de investigación: Contaduría: información, control e impacto social. Correo electrónico: juanlezama@usantotomas.edu.co ORCID: https://orcid.org/0000-0002-6009-7022

4 Contador público, magíster en Ciencias Económicas, international master executive MBA, Fundesem Business School. Docente de la Facultad de Contaduría Pública, Universidad Santo Tomás, Bogotá D. C., Colombia. Miembro del grupo de investigación: Contaduría: información, control e impacto social. Correo electrónico: jorge.garciac@usantotomas.edu.co - ORCID: https://orcid.org/0000-0003-1248-1094 


\title{
Resumen
}

El objetivo de este artículo fue proponer y aplicar un método de valoración para las pymes en Colombia. Se diseñó una metodología de valoración basada en el flujo de caja y se utilizó en las empresas Colombiana de Carnes CJC S. A. S., Comercial Química Ltda. y Distrimarcas S. A. S. con información del 2002 al 2016. En las empresas objeto de estudio se realizó un análisis estratégico para analizar los factores de éxito y los riesgos que tiene el sector, conjuntamente se evaluó su información contable y financiera para finalmente realizar las proyecciones necesarias para determinar el valor intrínseco de las pymes. Se encontró que la valoración es mayor al valor de los activos en las empresas de estudio, a excepción de CJC S. A. S. que registra un valor menor debido a que sus flujos de caja libre han disminuido por problemas en la administración de sus recursos operacionales. Este modelo de valoración demuestra que es consistente con la información financiera y con la situación de la empresa por lo que es adecuado para aplicarlo en la mayoría de las pymes en Colombia realizando los ajustes pertinentes al sector y particularidades de cada una.

Palabras clave: pymes, valoración, flujo de caja libre, WACC, costo de capital.

\section{Proposal for the valuation of SMEs in Colombia. A case study in the commercial and industrial sector}

\begin{abstract}
The objective of this article is to propose and apply a valuation method for SMEs in Colombia. A valuation methodology was designed based on the cash flow and was used in the Colombian companies of Carnes CJC S. A. S., Comercial Química Ltda. and Distrimarcas S. A. S. with information from 2002 to 2016. In the companies under study, a strategic analysis was carried
\end{abstract}


out to analyze the success factors and risks of the sector, together with the evaluation of their accounting and financial information to finally make the necessary projections to determine the intrinsic value of SMEs. It was found that the valuation is greater than the value of the assets in the study companies except for CJC S. A. S., which registers a lower value because its free cash flows have decreased due to problems in the management of its operational resources. This valuation model shows that it is consistent with the financial information and the situation of the company, so it is appropriate to apply it in the majority of SMEs in Colombia making the relevant adjustments to the sector and particularities of each.

Keywords: SMEs, valuation of companies, free cash flow, WACC, cost of capital.

\section{Introducción}

Las finanzas corporativas, para brindar una herramienta que permita tomar decisiones gerenciales y de financiación, han desarrollado diferentes teorías alrededor de la valoración de empresas que le permiten al inversionista y a la gerencia tener distintos panoramas sobre la situación financiera y las necesidades de financiamiento a corto y largo plazo.

Para la valoración de empresas uno de los aspectos a tener en cuenta es la capacidad de generación de efectivo (Damodaran, 2002; Fernández, 2008), pero además, es necesario saber el objetivo por el cual se está realizando, el tamaño, la trayectoria, la continuidad, el sector y entorno económico y la calidad de información (Moscoso y Botero, 2013).

Dentro de las metodologías para determinar el valor de la empresa, se encuentran aquellas basadas en la información financiera suministrada en el estado de situación financiera o balance general como los métodos de valor contable, valor en libros ajustados, valor de liquidación y valor sustancial; al igual que la extraída a partir del estado de resultados como 
el método basado en el múltiplo PER, el valor de los dividendos y el de múltiplos o ratios financieros.

Igualmente se emplea el método basado en el good will que utiliza cifras contables y los intangibles que generan valor para la compañía, los métodos basados en descuentos de flujo y aquellos fundamentados en opciones reales.

En las empresas que cotizan en la bolsa de valores, el precio de las acciones en el mercado sirve como referente para determinar el valor de la empresa; por el contrario, en las pymes o en aquellas que cuentan con un capital diferente al accionario, no tienen definido un valor de mercado y por lo tanto deben utilizar algún método de valoración.

De acuerdo con Confecámaras (2015), el 75 \% de las empresas en Colombia son pymes, por lo que la mayoría de estas no tienen identificado su valor; debido a esto, en esta investigación se tuvo como objetivo proponer una metodología para la valoración de las pymes acorde con el contexto colombiano. Para esto se analizan los diversos métodos de valoración y se determina el que es más pertinente a las condiciones de las pymes que tengan intención de continuidad y una trayectoria en el mercado.

Para el desarrollo de esta investigación se revisaron los diferentes métodos de valoración con el fin de identificar el más adecuado y posteriormente se realizó un análisis estratégico, contable y financiero y prospectivo del sector pyme. Finalmente, se aplicó el método escogido a tres pymes que tienen un reconocimiento, trayectoria, valor de ingresos considerable y que cuentan con la información suficiente para poder validar la aplicabilidad y la viabilidad de la metodología en ellas.

\section{Revisión de literatura de la valoración de empresas}

Poder cuantificar la empresa permite identificar con rigurosidad la viabilidad económica de la misma, revisar si las perspectivas en el futuro son las 
adecuadas y si las inversiones realizadas cumplen con las expectativas del inversionista, los cuales son objetivos en las finanzas corporativas.

El principal objetivo de la administración financiera es la maximización del valor de la empresa, el cual es un concepto diferente a la creación de utilidad. Por una parte, la creación de valor hace referencia al crecimiento financiero que tiene la empresa a través del tiempo, y la creación de la utilidad se refiere a la maximización de la riqueza de los inversionistas (Weston y Copeland, 1994, p. 5).

La maximización de valor de un negocio implica tener en cuenta el valor del dinero en el tiempo, el grado de riesgo de la corriente de ingresos, la calidad de los flujos de caja, la responsabilidad social y los factores externos que afectan la continuidad y crecimiento de la empresa (Weston y Copeland, 1994).

En principio, la valoración se puede entender como el proceso por el cual se determina la cantidad a pagar para invertir en la empresa (Rosenberg, 2001); igualmente, la valoración corresponde a la estimación del valor justificable para el capital de una compañía (Noussan, 2012).

De acuerdo con Parra (2013), la valoración es el

[...] proceso mediante el cual se asignan cifras a eventos o hechos económicos, de acuerdo con reglas, con unos propósitos particulares para dar a conocer al inversionista la recuperación de su capital en el presente, considerando unas variables que permitan determinar su valor en el tiempo. (P. 86).

La importancia de determinar el valor de una empresa cambia dependiendo de los intereses de cada usuario, particularmente, para los inversionistas es útil analizar si la inversión realizada corresponde a sus expectativas, identificar el valor a pagar por la adquisición de activos e incluso calcular el capital a recuperar en una empresa en proceso de liquidación. 
Así mismo, dentro de los propósitos por los cuales es necesario valorar la empresa, se encuentran: fijar el precio de negociación de una empresa; determinar los productos o servicios que se puede mantener o prescindir; poder comparar el valor con el mercado; justificar el precio en la colocación de nuevas acciones; identificar los generadores de valor; tomar decisiones sobre el portafolio de negocio, su continuidad y expansión; por cuestiones de herencias y para cuantificar la creación del valor de la empresa y sus impactos (Fernández, 2004).

Cuantificar la empresa depende del tipo y sector de la empresa, al igual que la razón por la cual se está realizando la valoración, debido a que no es lo mismo hacer una valoración de una empresa en liquidación que pretende ser vendida o recuperar los activos disponibles, a una con intención de negocio en marcha en el que se pretende identificar el potencial que tiene la empresa en un futuro. De igual forma, la medición depende de la forma en que se valore la empresa; en la Tabla 1 se pueden apreciar algunos de los métodos existentes:

Tabla 1. Métodos de valoración de empresas

\begin{tabular}{l|l}
$\begin{array}{l}\text { Agrupación de métodos de } \\
\text { valoración de empresas }\end{array}$ & \multicolumn{1}{c}{ Métodos de valoración de empresas } \\
\hline Balance & $\begin{array}{l}\text { Valor contable } \\
\text { Valor contable ajustado } \\
\text { Valor de reposición } \\
\text { Valor sustancial } \\
\text { Valor de liquidación } \\
\text { Valor de mercado }\end{array}$ \\
\hline Estado de resultados & Múltiplo de utilidad \\
& Múltiplo de ventas \\
& Múltiplo Ebitda \\
& Múltiplo precio-ganancia (PER) \\
& Múltiplo precio-ganancia con crecimiento (PEG) \\
& Otros múltiplos \\
\hline Good will o mixtos & Unión de expertos \\
& Contables europeos \\
& Renta abreviada \\
& Otros \\
\hline
\end{tabular}




\begin{tabular}{l|l}
\hline $\begin{array}{c}\text { Agrupación de métodos de } \\
\text { valoración de empresas }\end{array}$ & \multicolumn{1}{c}{ Métodos de valoración de empresas } \\
\hline Descuento de flujos de caja & $\begin{array}{l}\text { Flujo de caja libre } \\
\text { Flujo de caja de accionistas } \\
\text { Flujo de caja de capital } \\
\text { Valor de precio ajustado }\end{array}$ \\
\hline Creación de valor & $\begin{array}{l}\text { Valor económico agregado } \\
\text { Beneficio económico } \\
\text { Valor agregado de liquidez } \\
\text { Retorno sobre la inversión de los flujos de caja } \\
\text { Valor de mercado agregado }\end{array}$ \\
\hline Opciones & $\begin{array}{l}\text { Opciones reales } \\
\text { Black y Scholes }\end{array}$ \\
\hline
\end{tabular}

Fuente: tomado de Moscoso y Botero (2013, p. 247).

De acuerdo con la forma como es obtenida la información, esta se puede clasificar en cinco grupos, a saber: balance general, estado de resultados o ingresos, good will, flujo de caja descontado o creación de valor y opciones reales (Fernández, 2004). Cada una depende del objetivo y método sobre el cual se va a realizar la valoración de la empresa.

\section{Antecedentes de métodos de valoración en las pymes}

Las características de las empresas difieren de los contextos económicos, sociales y culturales de cada país, por esta razón, los métodos de valoración deben incluir el entorno externo para considerar las dinámicas propias de cada lugar. Considerando lo anterior, en los estudios revisados se han encontrado tres tipos de investigación con referencia a la valoración de empresas: valoración de empresas recién creadas, casos de valoración aplicados y estudios sobre valoración para casos específicos (modelo gerencial, sectores, pymes, etc.).

En las propuestas de medición de empresas recién creadas, es importante que la calidad de la información sea adecuada al tipo de empresa, entorno económico y el criterio del analista (Moscoso y Botero, 2013). 
Con respecto a la valoración de empresas aplicadas y casos específicos se evidencia que los modelos más utilizados para determinar el valor de las pymes se encuentran basados en el flujo de caja.

Narváez (2008) por medio del método de rendimientos determinó el valor de cuatro empresas del sector privado y tres públicas del Perú, e indica que:

El método del flujo de caja libre descontado resulta ser el más fiable y, en la práctica, es el más utilizado, pues se basa en la capacidad de generar ingresos futuros por la empresa en marcha, independientemente de que sea privada o pública. No obstante, sería recomendable complementarlo con un análisis comparativo basado en multiplicadores de empresas similares. (P. 30).

Teniendo en cuenta que es necesario seleccionar un método comprensible para la valoración de las pequeñas y medianas empresas, Rojo (2009) propone el modelo de estimación de los descuentos y el método de descuento de flujos de efectivo para identificar los porcentajes y tasas de descuento relacionado al riesgo específico asociado a la actividad de las empresas familiares españolas y así de esta forma evitar la subjetividad de su valoración.

De igual forma, Barrientos y Bedoya (2017) realizan la valoración por medio del método de flujo de caja descontado de una mediana empresa colombiana que aplica NIIF para pymes y evalúa los flujos de caja en riesgo bajo metodología CFar con la finalidad de integrar variables de incertidumbre y riesgo; este modelo ajustado para realizar la aplicación de las funciones de probabilidad para la proyección de variables mediante la simulación de Monte Carlo.

Con relación al método de flujos de caja proyectados, Gutiérrez, Asmay y Umañan (2011) identifican que esa es la mejor opción y determinan el valor de un hipódromo en Chile con la información financiera entre el 2003 y el 2008. Este modelo aplicado tiene restricciones debido a la reducción de los períodos y los riesgos operativos y financieros relacionados con los ciclos 
económicos de la industria y la economía, es decir, que se ve afectado por variables exógenas no incluidas dentro del cálculo utilizado.

La inclusión del riesgo es necesaria para la determinación del valor de una mediana empresa debido a que podrían aplicarse modelos que no correspondan a las necesidades del entorno y de los inversionistas; esta situación se ve reflejada en pymes del sector transportista de Maracaibo (Venezuela), las cuales utilizan técnicas de valoración de tipo histórico basadas en los principios de liquidez y rendimiento, los cuales no resultan adecuados para implementar métodos dinámicos basados en los principios financieros (Caicedo y Angulo, 2017).

Igualmente, se utilizaron métodos de valoración de flujo de caja libre y utilidad económica que se acercan a un modelo gerencial de valor (Correa, Jaramillo, Ramírez y Castaño, 2008) y la metodología basada en el valor económico agregado, pensada para un análisis a largo plazo para medir la situación financiera de las empresas pymes de cualquier sector (Escobar, Arias y Portilla, 2009).

\section{Metodología}

El propósito de la investigación fue diseñar una propuesta para valorar pymes en Colombia que tengan trayectoria e intención de continuidad, por lo tanto, la valoración se realizó desde el punto de vista de un inversionista externo que quiere invertir en la empresa y no tiene acceso a información protegida y de uso interno de esta.

Para el desarrollo de esta investigación se evaluaron los métodos de valoración de empresas y a partir de estas, se construyó la metodología de valoración que más se ajustara a las pymes de Colombia de acuerdo con su entorno, aplicabilidad y antecedentes de valoración.

Luego, se aplicó el método de valoración a las pymes Colombiana de Carnes CJC S. A. S., Comercial Química Ltda. y Distrimarcas S. A. S., las 
cuales se seleccionaron por tener reconocimiento y trayectoria en el país. La información financiera de las empresas fue obtenida de la Supersociedades para los períodos 2012 al 2016.

Para la aplicación de la metodología se siguieron los lineamientos planteados por Palepu (2010) que tiene en cuenta las cuatro fases que un inversionista debe analizar sobre una empresa en marcha para realizar la valoración.

La primera fase es el análisis estratégico del negocio, que tiene como propósito identificar los factores clave de éxito y los riesgos clave del negocio, evaluar el potencial de ganancia a nivel cualitativo.

La segunda fase es el análisis contable que tiene como propósito evaluar el grado en que la contabilidad capta la realidad de la empresa, es decir, identificar los puntos en los que la contabilidad es flexible, la idoneidad y relación entre las políticas y las estimaciones contables.

La tercera fase es el análisis financiero de cada empresa objeto de estudio, el cual, consiste en hacer uso de datos financieros para evaluar el rendimiento actual e histórico de una organización, al igual que su sostenibilidad financiera.

La cuarta y última fase es el análisis prospectivo que se centra en la provisión del futuro de una organización; en este aspecto se proyectan los estados financieros y se hace la valoración respectiva. En esta última etapa, se sintetiza el análisis estratégico, contable y el análisis financiero para determinar el valor intrínseco de la organización.

\section{Selección del método de valoración}

En la Tabla 2 se describe cada uno de los métodos de valoración y se identifican las ventajas y desventajas de cada uno con respecto a su aplicación en empresas pymes colombianas. 
Tabla 2. Características de los métodos de valoración de empresas

\begin{tabular}{|c|c|c|c|}
\hline $\begin{array}{l}\text { Método de } \\
\text { valoración }\end{array}$ & Descripción & Ventajas & Desventajas \\
\hline \multicolumn{4}{|c|}{$\begin{array}{c}\text { Métodos basados en el balance general: se valora la empresa de acuerdo con la } \\
\text { estimación del valor de los activos. }\end{array}$} \\
\hline $\begin{array}{l}\text { Método } \\
\text { de valor } \\
\text { contable }\end{array}$ & $\begin{array}{l}\text { Hace uso de la ecua- } \\
\text { ción contable: } \\
\text { eliminando partidas } \\
\text { que no implican salidas } \\
\text { de dinero (provisiones, } \\
\text { depreciación, otras). }\end{array}$ & $\begin{array}{l}\text { Facilidad de obtener } \\
\text { la información. } \\
\text { Rápido de valorar. }\end{array}$ & $\begin{array}{l}\text { Las partidas no siem- } \\
\text { pre se hacen a valor de } \\
\text { mercado. } \\
\text { No tiene en cuenta } \\
\text { las expectativas de la } \\
\text { empresa. } \\
\text { No tiene en cuenta } \\
\text { el entorno interno y } \\
\text { externo. } \\
\text { No considera el riesgo. }\end{array}$ \\
\hline $\begin{array}{l}\text { Método } \\
\text { de valor } \\
\text { en libros } \\
\text { ajustado }\end{array}$ & $\begin{array}{l}\text { Igual al anterior, } \\
\text { solo que los activos y } \\
\text { pasivos son ajustados } \\
\text { al valor estimado del } \\
\text { mercado ya sea al valor } \\
\text { de reposición o de } \\
\text { realización (Jaramillo, } \\
\text { 2010, p. 216). }\end{array}$ & $\begin{array}{l}\text { Usa el valor de } \\
\text { mercado en la } \\
\text { valoración. } \\
\text { Facilidad de obtener } \\
\text { la información. }\end{array}$ & $\begin{array}{l}\text { Activos que no tienen } \\
\text { un valor claro de } \\
\text { mercado. } \\
\text { Presenta dificultades } \\
\text { cuando los últimos } \\
\text { años genere pérdidas o } \\
\text { es intermitente en los } \\
\text { resultados (Jaramillo, } \\
\text { 2010). } \\
\text { No tiene en cuenta } \\
\text { las expectativas de la } \\
\text { empresa. } \\
\text { No tiene en cuenta } \\
\text { el entorno interno y } \\
\text { externo. } \\
\text { Algunas partidas la } \\
\text { valoración dependen } \\
\text { del juicio profesional. } \\
\text { No considera el riesgo. }\end{array}$ \\
\hline
\end{tabular}




\begin{tabular}{|c|c|c|c|}
\hline $\begin{array}{l}\text { Método de } \\
\text { valoración }\end{array}$ & Descripción & Ventajas & Desventajas \\
\hline $\begin{array}{l}\text { Método de } \\
\text { valor de } \\
\text { liquidación }\end{array}$ & $\begin{array}{l}\text { Se basa en el valor } \\
\text { por el cual pueden ser } \\
\text { negociados los activos } \\
\text { y pasivos. }\end{array}$ & $\begin{array}{l}\text { Usa el valor de } \\
\text { mercado en la } \\
\text { valoración. }\end{array}$ & $\begin{array}{l}\text { Se usa para empresa } \\
\text { que están en pro- } \\
\text { ceso de liquidación } \\
\text { o suspensión de } \\
\text { actividades. } \\
\text { En algunas partidas la } \\
\text { valoración depende del } \\
\text { juicio profesional. } \\
\text { No tiene en cuenta } \\
\text { las expectativas de la } \\
\text { empresa. } \\
\text { No tiene en cuenta } \\
\text { el entorno interno y } \\
\text { externo. } \\
\text { No considera el riesgo. }\end{array}$ \\
\hline $\begin{array}{l}\text { Método } \\
\text { de valor } \\
\text { sustancial }\end{array}$ & $\begin{array}{l}\text { Asume que la com- } \\
\text { pañía continúa su } \\
\text { negocio en marcha. Lo } \\
\text { que hace este método } \\
\text { es medir los activos a } \\
\text { su valor de reposición. }\end{array}$ & $\begin{array}{l}\text { Usa el valor de } \\
\text { mercado en la } \\
\text { valoración. }\end{array}$ & $\begin{array}{l}\text { Se usa para empresas } \\
\text { que tienen baja renta- } \\
\text { bilidad, por finaliza- } \\
\text { ción de concesiones } \\
\text { administrativas o } \\
\text { por agotamiento del } \\
\text { recurso natural que se } \\
\text { está explotando. } \\
\text { En algunas partidas la } \\
\text { valoración depende del } \\
\text { juicio profesional } \\
\text { No tiene en cuenta } \\
\text { las expectativas de la } \\
\text { empresa. } \\
\text { No tiene en cuenta } \\
\text { el entorno interno y } \\
\text { externo. } \\
\text { No considera el riesgo. }\end{array}$ \\
\hline
\end{tabular}




\begin{tabular}{l|l|l|l}
\hline $\begin{array}{l}\text { Método de } \\
\text { valoración }\end{array}$ & Descripción & Ventajas & Desventajas \\
\hline
\end{tabular}

Método basado en el estado de resultados: en este grupo se determina el valor de la empresa por el tamaño de las ganancias, ventas, capacidad de producción u otros indicadores financieros.

\begin{tabular}{|c|c|c|c|}
\hline $\begin{array}{l}\text { Método } \\
\text { basado en } \\
\text { el múltiplo } \\
\text { PER }\end{array}$ & \begin{tabular}{l} 
Este método permite \\
analizar si la empresa \\
está sobrevalorada \\
o infravalorada al \\
contrastar el PER con \\
"la rentabilidad exigida \\
por los accionistas y \\
el crecimiento medio \\
estimado para el cash \\
flow generado por la \\
empresa" (Fernández, \\
2008) Iu00bfpor qu\} $\\
{\text { u00e9 se estlu00e1 }} \\
{\text { haciendo la valoraci }} \\
{\text { u00f3n de determinada }} \\
{\text { manera? y lu00bfpara }} \\
{\text { qulu00e9 y para qui\} } \\
{\text { u00e9n se estlu00e1 }} \\
{\text { haciendo la valoraci }} \\
{\text { u00f3n? Casi todos los }} \\
{\text { errores en valoraci\} } \\
{\text { u00f3n se deben a no }} \\
{\text { contestar adecuada- }} \\
{\text { mente a alguna de }} \\
{\text { estas preguntas, esto }} \\
{\text { es, a falta de conoci- }} \\
{\text { mientos o a falta de }} \\
{\text { sentido comlu00fan (o }} \\
{\text { a la falta de ambos. }}\end{array}} &{\begin{array}{l}\text { Tiene en cuenta las } \\
\text { expectativas de la } \\
\text { empresa al medir el } \\
\text { precio de mercado. } \\
\text { Usa el valor de } \\
\text { mercado en la } \\
\text { valoración. } \\
\text { Facilidad de obtener } \\
\text { la información. }\end{array}} &{\begin{array}{l}\text { Se requiere que la } \\
\text { empresa cotice en la } \\
\text { bolsa de valores. } \\
\text { No diferencia las } \\
\text { utilidades de la } \\
\text { operación con las no } \\
\text { operacionales. } \\
\text { En las utilidades } \\
\text { incluye partidas que } \\
\text { no implican una salida } \\
\text { o entrada de dinero. } \\
\text { La valoración no cuan- } \\
\text { tifica a la empresa, } \\
\text { pero sí indica si está } \\
\text { bien o mal valorada. }\end{array}} \\
\hline\end{array}$
\end{tabular}
\end{tabular}




\begin{tabular}{|c|c|c|c|}
\hline $\begin{array}{l}\text { Método de } \\
\text { valoración }\end{array}$ & Descripción & Ventajas & Desventajas \\
\hline $\begin{array}{l}\text { Método del } \\
\text { valor de los } \\
\text { dividendos } \\
\text { (basado } \\
\text { en los } \\
\text { postulados } \\
\text { de Gordon } \\
(1962) \text { ) }\end{array}$ & $\begin{array}{l}\text { El valor de la empresa } \\
\text { es representado como } \\
\text { el valor presente neto } \\
\text { de los dividendos } \\
\text { futuros descontando } \\
\text { la tasa requerida del } \\
\text { patrimonio; algunos } \\
\text { casos incluyen una } \\
\text { tasa de crecimiento } \\
\text { constante. }\end{array}$ & $\begin{array}{l}\text { Tiene en cuenta el } \\
\text { valor del dinero en } \\
\text { el tiempo. } \\
\text { Considera el riesgo } \\
\text { al determinar una } \\
\text { tasa requerida del } \\
\text { inversionista. }\end{array}$ & $\begin{array}{l}\text { Se requiere que la } \\
\text { empresa cotice en la } \\
\text { bolsa de valores. } \\
\text { No todas las empre- } \\
\text { sas exitosas reparten } \\
\text { dividendos. } \\
\text { Los retornos se cuan- } \\
\text { tifican solo por los } \\
\text { dividendos y no por la } \\
\text { capacidad de la empre- } \\
\text { sa de generar valor. } \\
\text { No tiene en cuenta el } \\
\text { entorno interno de la } \\
\text { empresa }\end{array}$ \\
\hline $\begin{array}{l}\text { Método de } \\
\text { múltiplos } \\
\text { o ratios } \\
\text { financieros }\end{array}$ & $\begin{array}{l}\text { Utiliza indicadores } \\
\text { financieros para } \\
\text { determinar el valor de } \\
\text { la compañía. } \\
\text { Otra forma es compa- } \\
\text { rando algunas ratios } \\
\text { con otras empresas } \\
\text { para establecer su } \\
\text { valor. }\end{array}$ & $\begin{array}{l}\text { Facilidad de obtener } \\
\text { la información. } \\
\text { Rápido de valorar. } \\
\text { Se compara con el } \\
\text { sector. }\end{array}$ & $\begin{array}{l}\text { Se utiliza en empresas } \\
\text { que cotizan en bolsa. } \\
\text { La valoración no cuan- } \\
\text { tifica a la empresa, } \\
\text { pero sí indica si está } \\
\text { bien o mal valorada. } \\
\text { No tiene en cuenta } \\
\text { las expectativas de la } \\
\text { empresa ya que se basa } \\
\text { en datos históricos. } \\
\text { No tiene en cuenta el } \\
\text { entorno interno de la } \\
\text { empresa. }\end{array}$ \\
\hline
\end{tabular}




\begin{tabular}{l|l|l|l}
\hline $\begin{array}{l}\text { Método de } \\
\text { valoración }\end{array}$ & Descripción & Ventajas & Desventajas \\
\hline
\end{tabular}

El método basado en el good will o mixto: consiste en una valoración de carácter mixto, el cual no solo utiliza las cifras de los activos que cuenta o los ingresos que genera, sino también aquellos intangibles que contribuyen en la generación del valor.

\begin{tabular}{|c|c|c|c|}
\hline $\begin{array}{l}\text { El método } \\
\text { clásico }\end{array}$ & $\begin{array}{l}\text { El método clásico de } \\
\text { valoración se expresa } \\
\text { como: } \\
\text { Donde } \\
\text { O en algunos casos, se } \\
\text { usa también: } \\
\text { Donde }\end{array}$ & $\begin{array}{l}\text { Facilidad de obtener } \\
\text { la información. } \\
\text { Rápido de valorar. } \\
\text { Es un método de } \\
\text { dominio público } \\
\text { debido a que es } \\
\text { una metodología } \\
\text { de creación de } \\
\text { valor que se puede } \\
\text { utilizar libremente } \\
\text { a diferencia de } \\
\text { otros como el Stern } \\
\text { Stewart Et CO y } \\
\text { el EVA que tiene } \\
\text { un costo por su } \\
\text { utilización (Vidarte, } \\
\text { 2009). }\end{array}$ & $\begin{array}{l}\text { Las expectativas de la } \\
\text { empresa se basan en } \\
\text { datos históricos y en el } \\
\text { coeficiente. } \\
\text { Poco uso empresarial. }\end{array}$ \\
\hline
\end{tabular}

Métodos basados en descuentos de flujos: determina el valor de la empresa por medio del valor presente neto de los flujos de caja, utilidad o valor que generará la empresa, descontándolo a una tasa que incluye el riesgo de los flujos.

\begin{tabular}{|c|c|c|c|}
\hline $\begin{array}{l}\text { Método de } \\
\text { flujos de } \\
\text { utilidades } \\
\text { descontadas }\end{array}$ & $\begin{array}{l}\text { Determina el valor de } \\
\text { la empresa por medio } \\
\text { del valor presente de } \\
\text { las utilidades futuras } \\
\text { descontando una tasa } \\
\text { de descuento. }\end{array}$ & $\begin{array}{l}\text { Facilidad de obtener } \\
\text { la información. } \\
\text { Soporte teórico }\end{array}$ & $\begin{array}{l}\text { No diferencia las } \\
\text { utilidades de la } \\
\text { operación con las no } \\
\text { operacionales. } \\
\text { En las utilidades } \\
\text { incluye partidas que } \\
\text { no implican una salida } \\
\text { o entrada de dinero. }\end{array}$ \\
\hline $\begin{array}{l}\text { Método } \\
\text { de flujo } \\
\text { de fondos } \\
\text { descontados }\end{array}$ & $\begin{array}{l}\text { Determina el valor de } \\
\text { la empresa por el valor } \\
\text { presente de la genera- } \\
\text { ción interna de fondos } \\
\text { proyectados: utilidad } \\
\text { neta + depreciación y } \\
\text { otras partidas que no } \\
\text { implican salidas de } \\
\text { efectivo. }\end{array}$ & $\begin{array}{l}\text { Facilidad de obtener } \\
\text { la información. } \\
\text { Soporte teórico }\end{array}$ & $\begin{array}{l}\text { No diferencia las } \\
\text { utilidades de la } \\
\text { operación con las no } \\
\text { operacionales. } \\
\text { No considera los acti- } \\
\text { vos no corrientes que } \\
\text { están relacionados con } \\
\text { la operación. }\end{array}$ \\
\hline
\end{tabular}




\begin{tabular}{|c|c|c|c|}
\hline $\begin{array}{l}\text { Método de } \\
\text { valoración }\end{array}$ & Descripción & Ventajas & Desventajas \\
\hline $\begin{array}{l}\text { Método } \\
\text { de flujo de } \\
\text { efectivo }\end{array}$ & $\begin{array}{l}\text { Es similar a la anterior } \\
\text { solo que este método } \\
\text { detalla los fondos en } \\
\text { inversión, operación } \\
\text { y financiación; da } \\
\text { más importancia a } \\
\text { los fondos generados } \\
\text { por las actividades de } \\
\text { operación. }\end{array}$ & $\begin{array}{l}\text { Facilidad de obtener } \\
\text { la información. } \\
\text { Soporte teórico. }\end{array}$ & $\begin{array}{l}\text { No diferencia las } \\
\text { utilidades de la } \\
\text { operación con las no } \\
\text { operacionales. } \\
\text { No considera los acti- } \\
\text { vos no corrientes que } \\
\text { están relacionados con } \\
\text { la operación. }\end{array}$ \\
\hline $\begin{array}{l}\text { Método de } \\
\text { flujo de caja } \\
\text { libre }\end{array}$ & $\begin{array}{l}\text { Para obtener los flujos } \\
\text { de caja libre se toman } \\
\text { las actividades de ope- } \\
\text { ración y de financia- } \\
\text { ción, de igual forma los } \\
\text { flujos de los activos no } \\
\text { corrientes relacionados } \\
\text { con la operación y se } \\
\text { elimina de la utilidad } \\
\text { del ejercicio lo que } \\
\text { no sea operacional, la } \\
\text { idea es evidenciar lo } \\
\text { que puede producir la } \\
\text { empresa por sí misma. }\end{array}$ & $\begin{array}{l}\text { Facilidad de obtener } \\
\text { la información. } \\
\text { Soporte teórico. } \\
\text { Útil para empresas } \\
\text { con alto apalanca- } \\
\text { miento financiero. }\end{array}$ & $\begin{array}{l}\text { Dificultad en la esti- } \\
\text { mación del costo de } \\
\text { capital. }\end{array}$ \\
\hline $\begin{array}{l}\text { Métodos } \\
\text { basados en } \\
\text { opciones } \\
\text { reales }\end{array}$ & $\begin{array}{l}\text { Se aplica el modelo de } \\
\text { valoración binominal } \\
\text { propuesto por Cox, } \\
\text { Ross, y Rubinstein } \\
\text { (1979) o el modelo } \\
\text { de valoración Black y } \\
\text { Scholes (1973). }\end{array}$ & $\begin{array}{l}\text { Soporte teórico. } \\
\text { Considera el riesgo. } \\
\text { Considera las } \\
\text { expectativas de la } \\
\text { empresa. }\end{array}$ & $\begin{array}{l}\text { Modelo teórico con } \\
\text { poco uso empresarial. } \\
\text { La formulación resulta } \\
\text { compleja para empre- } \\
\text { sas pymes. }\end{array}$ \\
\hline
\end{tabular}

Fuente: elaboración propia.

De acuerdo con los datos anteriores, el método basado en el good will y el basado en descuento de flujos son los más apropiados para aplicar en las pymes; sin embargo, los métodos basados en descuento de flujos son los más completos y los más utilizados para la valoración, debido a su rigurosidad, capacidad para determinar los factores que crean o destruye valor y para reconocer el cambio de valor en el futuro (Jaramillo, 2010; Narváez Liceras, 2009). 
Considerando lo anterior y los antecedentes sobre el tema, el método de flujo de caja libre es el que se sugiere para aplicar en las pymes en Colombia ya que es útil para las empresas que tienen un alto apalancamiento financiero y se valora la empresa por los fondos que quedan después de cumplir con las obligaciones financieras, cubrir los gastos de capital y las necesidades de capital de trabajo (Damodaran, 2002).

\section{Construcción de la metodología}

Las pymes poseen una mayor incertidumbre en el tema de la valoración al no contar con un mercado de valores que permita hacerles seguimiento, además, en Colombia solo están obligadas a informar los estados financieros anuales y no dan una información específica como es el caso de las revelaciones, las cuales son importantes para el negocio y para los usuarios de la información.

Para el desarrollo de la investigación se partió de la información disponible como los estados financieros históricos y estudio del sector para realizar los pronósticos de los flujos de caja, además, se realizaron algunas estimaciones en información que no se tuvo disponible. A continuación, se detalla la metodología que se propone para valorar las empresas pymes en Colombia.

El modelo de valoración de la empresa por el método del flujo de caja libre, de acuerdo con Damodaran (2002) se define como:

$$
\text { Valor de la empresa }=\sum_{t=1}^{n} \frac{F C L_{t}}{\left(1+W A C C_{t}\right)^{t}}+\frac{\frac{F C L_{n+1}}{W A C C-g_{n}}}{\left(1+W A C C_{t}\right)^{n}}
$$

Donde:

$$
\begin{aligned}
& F C L_{t}=\text { Flujo de caja libre de la empresa en el año } t \\
& \text { WACC }=\text { Costo de capital promedio ponderado } \\
& g_{n}=\text { Tasa de crecimiento estable de la empresa } \\
& n=\text { período de tiempo a evaluar }
\end{aligned}
$$


El período para evaluar es el tiempo en el que se va a realizar el análisis de valoración, en donde existen tres etapas de análisis (Jaramillo, 2010): la primera es la fase de base, la cual parte de una fecha reciente, principalmente de la que se cuente con información. La segunda es la de pronóstico, en la que normalmente se toman entre 5 y 10 años, pero esto es relativo dependiendo del tipo del negocio y su planeación al corto o mediano plazo. Por último, la continua, que es el período que se toma posterior a la del pronóstico, en donde se utiliza la hipótesis de negocio en marcha.

Para estimar el flujo de caja libre en cada período se determina de la siguiente manera:

$$
F C L=E B I T(1-\text { Tasa impositiva }) \cdot(1-\text { Tasa de reinversión })
$$

Donde:

$E B I T=$ Utilidad antes de intereses e impuestos EBIT(1-Tasa impositiva) tambien es conocido como NOPAT

Tasa de reinversión:

(-Depreación y amortización + Capital de trabajo neto + Reinversión de capital)/Ventas

De acuerdo con Damodaran (2002), la tasa de crecimiento de la empresa en el año $t$ se puede estimar de la siguiente manera:

$g_{t}=$ tasa de reinversión ROIC

Donde:

ROIC $=$ Retorno sobre el capital invertido

ROIC > WACC para garantizar que la empresa genere un crecimiento positivo.

Para estimar la tasa de crecimiento se usa el indicador del margen neto de la empresa; una vez obtenido este valor se puede calcular la tasa de reinversión como: 


$$
\text { tasa de reinversión } n_{t}=\frac{g_{t}}{R O I C_{t}}
$$

Donde:

$$
R O I C=\frac{E B I T(1-t)}{\text { Capital invertido }}
$$

Para determinar la utilidad antes de intereses e impuestos y la tasa de reinversión de capital, es necesario hacer una proyección de los estados financieros en los períodos de tiempo a evaluar. Las proyecciones se pueden realizar con un promedio histórico de crecimiento en ventas, costos y otras variables o con modelos econométricos; debido a la disponibilidad de la información para la aplicación de la metodología, se realiza con un promedio móvil basado en los datos históricos para el cálculo de la tasa de reinversión del capital, y, para la tasa de crecimiento estable, se realiza un promedio basado en los datos históricos.

Para determinar el costo de capital promedio ponderado o WACC en los períodos a analizar, se define como:

$$
W A C C_{t}=K_{e t} \% E_{t-1}+K_{d t}(1-t) \% D_{t-1}
$$

Donde:

$\% D_{t-1}$ relación deuda sobre activos y $\% E_{t-1}$ patrimonio sobre activos.

Al no contar con información detalla de los pasivos de las empresas de estudio, para la estimación del costo de la deuda $\left(K_{d}\right)$ se calculó el promedio geométrico de los gastos financieros sobre el total de la deuda de cada pyme.

Para determinar el costo de oportunidad del accionista sin endeudamiento y considerando que es una empresa que no cotiza en bolsa, se estima por medio del modelo $\mathrm{CAPM}^{5}$ ajustando las betas (Vélez, 2002):

$$
K_{u}=r_{f}+\beta_{u} \cdot\left(R_{M}-r_{f}\right)
$$

5

Capital Assets Pricing Model 
Donde:

$\mathrm{r}_{\mathrm{f}}=$ Tasa libre de riesgo

$\beta_{u}=$ Beta desapalancado de la empresa

$\left(R_{M}-r_{f}\right)=$ Prima por riesgo de mercado

Para la estimación de la beta de la empresa de acuerdo con Vélez Pareja (2002), se debe identificar:

El sector de la economía al cual pertenece la firma, calcular el costo del patrimonio sin deuda $(\mathrm{Ku})$ para un número apropiado de empresas del sector (depende de la disponibilidad de información), calcular un promedio ponderado (de acuerdo con las ventas o la utilidad operacional) del coeficiente beta sin deuda y apalancar o endeudar el coeficiente beta para la firma no transada. (P. 58).

Considerando que el mercado de valores de Colombia es pequeño, la información histórica es muy reciente ya que parte del año 2001 y se presentan dificultades en el cálculo por sector de las betas e incluso algunos no son significativos. Se utilizaron las betas del sector para países emergentes calculados por Damodaran (2018) para el año 2017. Una vez obtenidos los betas y siguiendo a Vélez (2002), se aplicó la siguiente ecuación para determinar la beta desapalancado de una empresa que no cotiza en la bolsa:

$$
\beta_{u}=\left[\frac{\beta_{\text {proxy }}}{1+\frac{D_{\text {proxy }}(1-t)}{E_{\text {proxy }}}}\right]
$$

Donde:

$\beta_{\text {proxy }}=$ Beta apalancado del sector

$\frac{D_{\text {proxy }} \cdot(1-t)}{E_{\text {proxy }}}=\begin{aligned} & \text { relación deuda sin el efecto del impuesto sobre } \\ & \text { patrimonio del sector }\end{aligned}$

$t=$ tasa de impuesto efectiva de la empresa 
Se determinó una tasa libre de riesgo del 5,87 \% efectivo anual por medio de un promedio geométrico de la rentabilidad de la canasta de los Títulos de Tesorería TES con vencimiento en julio del 2020, mayo del 2022, julio del 2024 y agosto del 2026, que corresponde a la información obtenida del portal financiero del grupo AVAL (2017). Además, para la prima por riesgo de mercado se utilizó el 5.08 \% efectivo anual calculado para Colombia (Damodaran, 2018).

Una vez obtenido y considerando que es constante se calcula el costo de oportunidad del accionista con endeudamiento, como dicen Harris y Pringle (1985) citados por Vélez-Pareja y Tham (2009):

$$
K_{e_{t}}=K_{u}+\left(k_{u}-k_{d_{t}}\right) \cdot(1-t) \frac{\% D_{t-1}}{\% E_{t-1}}
$$

Donde:

$K_{e t}=$ Costo de oportunidad del accionista con endeudamiento

$K_{u}=$ Costo de oportunidad del accionista sin endeudamiento

$\% D_{t-1}$ relación deuda sobre activos $y \% E_{t-1}$ patrimonio sobre activos.

\section{Aplicación de la metodología}

Las fases para valorar una empresa requieren conocimiento a un detalle mayor, tanto a nivel interno como externo para así tener una mejor estimación del valor de la empresa. No obstante, se presentan los resultados de la aplicación de la metodología basados en la información pública disponible de tres pymes en Colombia.

\section{Análisis estratégico}

Las pymes tienen un papel importante en la economía de varios países del mundo debido a que generan más empleo y tienen más poder de innovación que las entidades grandes, por tal motivo, las empresas grandes no se preocupan por crear, sino que más bien adquieren a este tipo de empresas (Velásquez, 2004). 
En Colombia la importancia de las pymes no se queda atrás, por ejemplo, en el año 2016 cerca del $95 \%$ de las empresas nuevas fueron micro y el $5 \%$ fueron pequeñas y medianas, las cuales generaron el $28 \%$ del PIB (Londoño y Sánchez, 2017). Igualmente, el primer resultado financiero bajo las NIIF colombianas de las pymes, de acuerdo con la Superintendencia de Sociedades (2017), indica que los activos y los ingresos aumentaron en el 2016 tal como se observa en la Tabla 3.

Tabla 3. Análisis financiero pymes (valores en millones de pesos)

\begin{tabular}{l|c|c|c}
\hline Rubro & $\mathbf{2 0 1 5}$ & $\mathbf{2 0 1 6}$ & Variación \\
\hline Activo & 66.16 & 73.77 & $11.49 \%$ \\
\hline Pasivo & 38.07 & 42.07 & $10.50 \%$ \\
\hline Patrimonio & 28.09 & 31.70 & $12.83 \%$ \\
\hline Ingresos operacionales & 93.86 & 107.86 & $14.91 \%$ \\
\hline Resultado del ejercicio & 2.27 & 4.14 & $82.27 \%$ \\
\hline
\end{tabular}

Fuente: elaboración propia con datos del informe de la Supersociedades (2017, p. 8).

Se debe tener en cuenta que según el informe de la Asociación Nacional de Instituciones Financieras (2017), el desempeño general y ventas que tuvieron las pymes colombianas para el primer semestre de 2017 fue catalogado como bueno, con una puntuación de 61 sobre 100 a pesar de tener una tendencia a la baja debido a la desaceleración de la economía del país.

Los datos fueron obtenidos a través de los reportes suministrados por la Superintendencia de Sociedades, acorde a la clasificación de pymes de la Ley 905 (2004). Se tomaron 3 empresas con activos de hasta 30000 millones pertenecientes al sector comercial e industrial y que presentan un alto nivel de ventas, las cuales son:

1. Comercial Química Ltda., dedicada al comercio al por mayor y al por menor de cosméticos, farmacéuticos, agroquímicos y veterinarios y se ubica en la ciudad de Bogotá; esta cuenta con una facturación a 2016 de 21275 millones de pesos. 
2. Colombiana de Carnes CJC S. A. S., se encarga del procesamiento y conservación de carne y productos cárnicos, con sede en la ciudad de Bogotá y reporta 21915 millones de pesos en facturación.

3. Distrimarcas S. A. S., comercializa productos alimenticios y de abarrotes en general, con sede en la ciudad de Itaguí y presenta ingresos por ventas de 20285 millones de pesos.

Como se puede observar, las empresas objeto de estudio pertenecen principalmente al sector de comercio al por mayor y al por menor, el cual es el tercer sector con mayor crecimiento de ingresos para el 2016, correspondiendo al $14.65 \%$ y antecedido por el sector agropecuario y manufacturero. De igual forma, las ganancias se incrementaron en un $83.11 \%$ entre el 2015 y el 2016, lo que indica que presenta una evolución en su economía este sector.

Igualmente, de acuerdo con el informe de la Superintendencia de Sociedades (2017), el sector comercial aumentó el $1.5 \%$ en su aporte al PIB para el 2016 en contraste con el 2015; por otro lado, las pymes proporcionaron el $44.95 \%$ del total de los ingresos generados para el 2016.

\section{Análisis contable y financiero}

En el año 2016 las empresas en Colombia presentaron un cambio de estándar contable surgido por la Ley 1314, para efecto las pymes pertenecientes al grupo 2 adquirieron la obligación de reportar la información financiera bajo las NIIF, este cambio afectó la contabilización de los estados financieros, acercándolas más a la realidad del mercado.

En la Tabla 4 se muestran los principales indicadores financieros para las empresas Colombiana de Carnes CJC S. A. S., Comercial Química Ltda. y Distrimarcas S. A. S. 


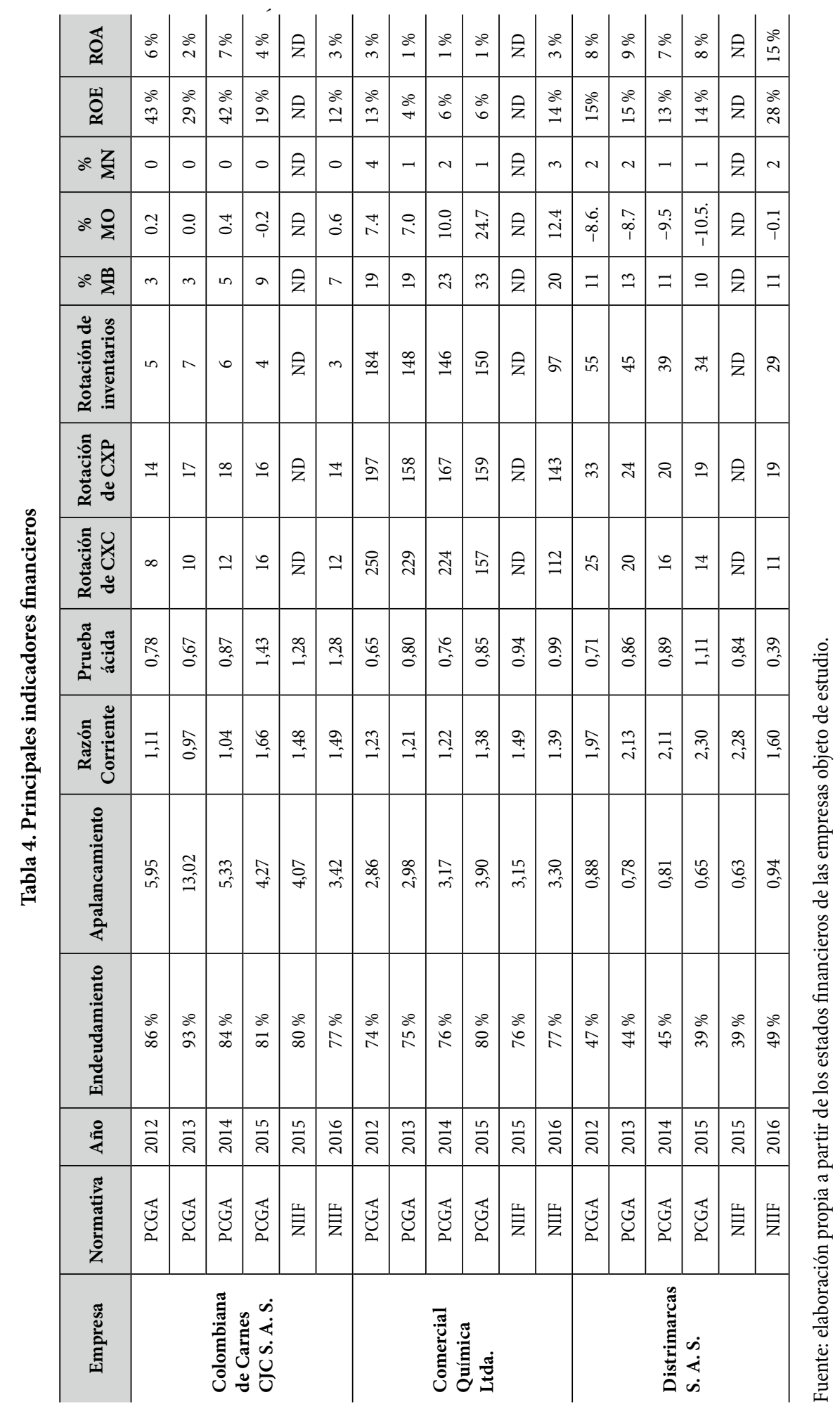


A continuación, se resaltan las observaciones más relevantes del análisis contable y financiero:

El endeudamiento para el 2016 de las empresas CJC S. A. S. y Comercial Química Ltda. es el que tiene mayor nivel, aunque con el cambio de estándar contable ha permitido que este disminuya. Respecto a Distrimarcas S. A. $S$. este indicador es menor, en principio porque corresponde a un sector diferente al de las otras empresas.

El indicador de apalancamiento financiero es más homogéneo en las primeras dos empresas, solamente en Distrimarcas S. A. S. el patrimonio supera el apalancamiento debido a que su financiación en su mayoría proviene de recursos propios. Con relación a la razón corriente en general para todas las empresas de estudio, esta es superior a 1, es decir, que las empresas cuentan con los activos suficientes para cubrir sus pasivos que deben cumplir a corto plazo.

La prueba ácida indica que las empresas CJC S. A. S. y Distrimarcas S. A. S. dependen en promedio de un $15 \%$ de los inventarios para solventar sus pasivos a corto plazo; su rotación de cartera, de cuentas por pagar y de inventarios es de 13, 18 y 12 días respectivamente. Por otra parte, Comercial Química Ltda. presenta una rotación en los indicadores mencionados de 186, 164 días y 142 días.

La empresa Comercial Química Ltda. presenta un margen bruto en promedio del $23 \%$, así mismo, el margen operacional se presenta constantemente positivo, el rendimiento del patrimonio en promedio es del 9 $\%$ y su rendimiento del activo total es del $9 \%$.

\section{Análisis prospectivo}

Con base en los datos históricos de las empresas se calcularon los flujos de caja libre de cada período y posteriormente se proyectaron a 5 años. En la Tabla 5 se muestran los resultados del flujo de caja histórico. Como se aprecia, la empresa CJC S. A. S. es la que tiene menor valor de FCL y la empresa Comercial Química Ltda. es la que mayor flujo de caja tiene. 


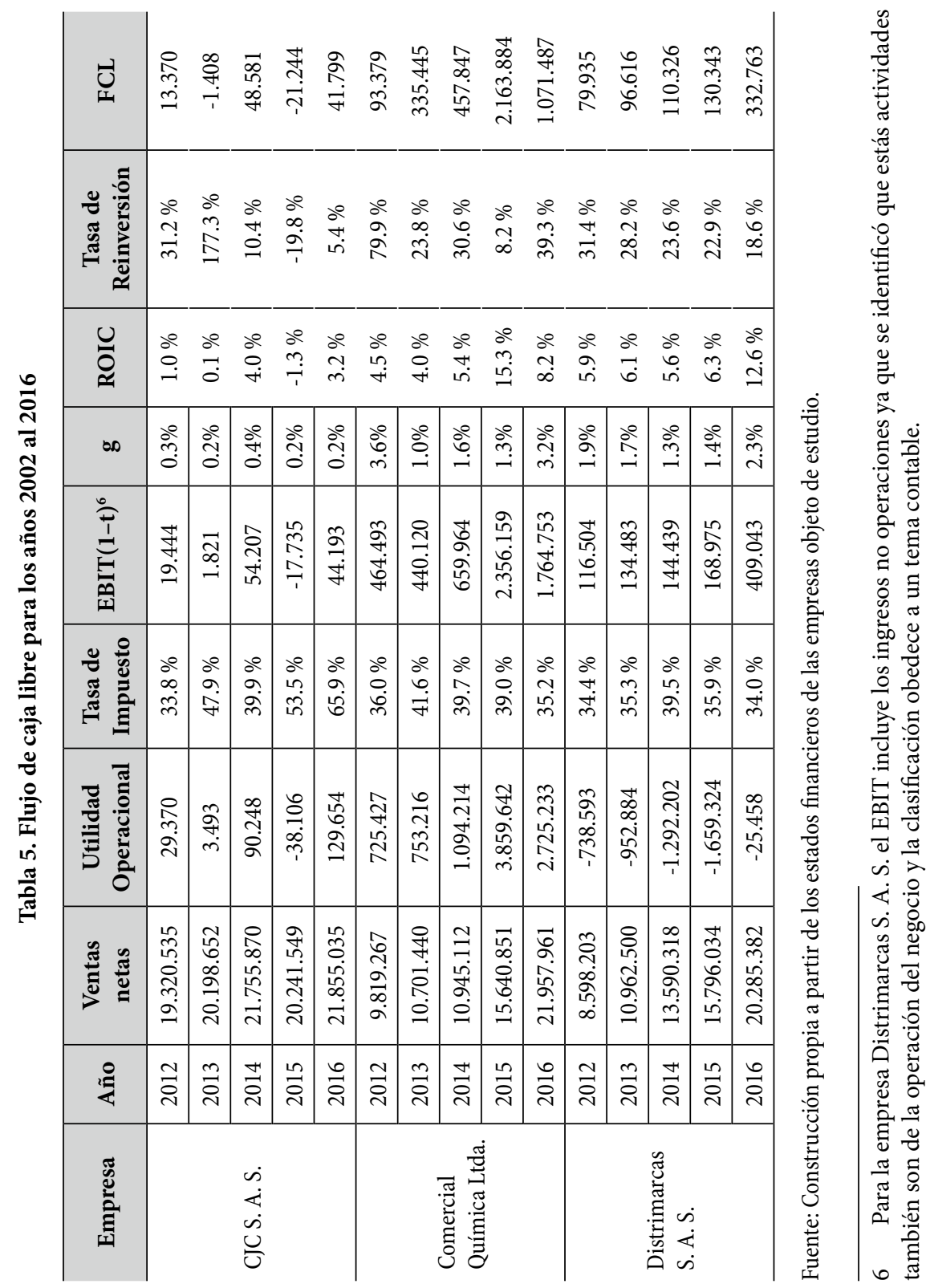


Para realizar la proyección de los flujos de caja libre, se utilizaron las ventas netas con una media móvil de tres años, a partir de este dato se calcularon las utilidades y los demás datos. Para la tasa de impuesto se tomó el promedio histórico debido a que las últimas reformas tributarias han hecho que los impuestos no sean estables, igualmente con la tasa de reinversión se hizo un promedio para que esta sea constante a través del tiempo. En la Tabla 6 se muestran los resultados del ejercicio. 


\begin{tabular}{|c|c|c|c|c|c|c|c|c|c|c|c|c|c|c|}
\hline ర્પ & 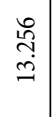 & 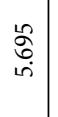 & 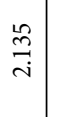 & 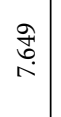 & $\begin{array}{c}\infty \\
\stackrel{+}{7} \\
\text { in }\end{array}$ & 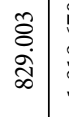 & 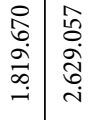 & 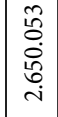 & 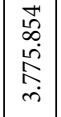 & $\begin{array}{l}\vec{b} \\
\stackrel{0}{0} \\
\stackrel{0}{0} \\
\end{array}$ & 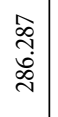 & $\begin{array}{l}8 \\
8 \\
0 \\
\infty \\
0 \\
n \\
m\end{array}$ & $\begin{array}{l}\stackrel{m}{\hat{N}} \\
\stackrel{i}{q}\end{array}$ & $\begin{array}{l}8 \\
\stackrel{8}{\circ} \\
\stackrel{2}{6}\end{array}$ \\
\hline 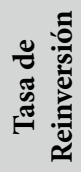 & $\begin{array}{l}\dot{\circ} \\
\stackrel{+}{+} \\
\dot{+}\end{array}$ & $\begin{array}{l}\dot{0} \\
\text { ò } \\
\text { 하 }\end{array}$ & $\begin{array}{l}\circ \\
\text { oे } \\
\text { fे }\end{array}$ & $\begin{array}{l}\dot{0} \\
\vdots \\
\dot{y}\end{array}$ & $\begin{array}{l}\dot{a} \\
\vdots \\
\vdots \\
\dot{q}\end{array}$ & $\begin{array}{l}0 \\
0 \\
+ \\
0 \\
0 \\
0\end{array}$ & 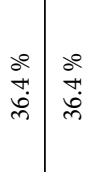 & 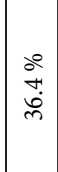 & 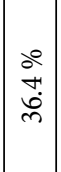 & $\begin{array}{l}\stackrel{0}{\circ} \\
\stackrel{2}{+} \\
\dot{N}\end{array}$ & $\begin{array}{l}\stackrel{0}{0} \\
\stackrel{+}{+}\end{array}$ & $\begin{array}{l}\stackrel{\circ}{0} \\
\stackrel{+}{+}\end{array}$ & $\begin{array}{l}\stackrel{0}{2} \\
\stackrel{\leftrightarrow}{\sim}\end{array}$ & $\begin{array}{l}\stackrel{\circ}{\circ} \\
\stackrel{+}{\mathrm{j}}\end{array}$ \\
\hline 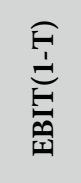 & $\begin{array}{l}\stackrel{+}{\oplus} \\
\stackrel{\sim}{+}\end{array}$ & 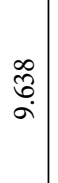 & $\begin{array}{l}\vec{t} \\
\dot{m}\end{array}$ & 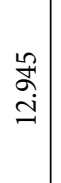 & $\begin{array}{l}\stackrel{n}{0} \\
\tilde{n} \\
\sigma\end{array}$ & 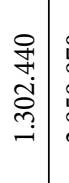 & 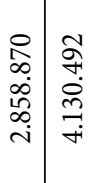 & 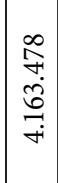 & 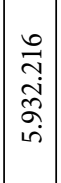 & $\left|\begin{array}{l}2 \\
\tilde{n} \\
\tilde{n} \\
\tilde{m}\end{array}\right|$ & $\begin{array}{l}\infty \\
\infty \\
m \\
\dot{m} \\
\infty \\
m\end{array}$ & $\begin{array}{l}\stackrel{2}{2} \\
\stackrel{2}{1} \\
\frac{1}{f}\end{array}$ & 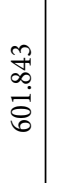 & 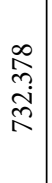 \\
\hline 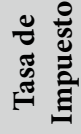 & $\begin{array}{c}\stackrel{0}{0} \\
\stackrel{1}{0} \\
\infty \\
+\end{array}$ & 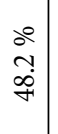 & $\begin{array}{l}0 \\
\sim \\
\infty \\
\dot{\phi}\end{array}$ & $\begin{array}{l}\partial^{0} \\
\sim \\
\infty \\
\dot{p}\end{array}$ & 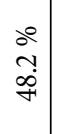 & $\begin{array}{l}0 \\
m \\
\infty \\
\infty\end{array}$ & \begin{tabular}{l|l}
$o$ & 0 \\
$m$ & $m$ \\
$\infty$ & $\infty$ \\
$m$ & $\infty$
\end{tabular} & $\begin{array}{l}0 \\
m \\
\infty \\
m\end{array}$ & $\mid \begin{array}{l}0 \\
0 \\
m \\
\infty \\
m\end{array}$ & $\begin{array}{l}0 \\
\infty \\
\infty \\
m\end{array}$ & $\begin{array}{l}0 \\
\infty \\
\infty \\
m\end{array}$ & $\begin{array}{l}\infty \\
\infty \\
\infty \\
m\end{array}$ & $\begin{array}{l}\stackrel{0}{ } \\
\infty \\
\dot{m}\end{array}$ & $\begin{array}{l}\circ \\
\infty \\
\dot{m} \\
\dot{m}\end{array}$ \\
\hline 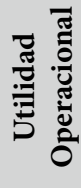 & $\begin{array}{l}\stackrel{2}{0} \\
m \\
\stackrel{+}{F}\end{array}$ & $\begin{array}{l}+ \\
\stackrel{0}{0} \\
\infty \\
0\end{array}$ & $\begin{array}{l}\stackrel{0}{0} \\
\hat{\sigma}\end{array}$ & $\begin{array}{l}\hat{\infty} \\
\stackrel{+}{+} \\
\dot{\sim}\end{array}$ & $\begin{array}{l}\overrightarrow{0} \\
\stackrel{0}{-}\end{array}$ & 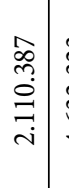 & 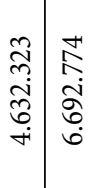 & 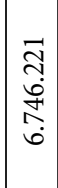 & 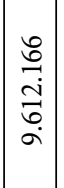 & $\begin{array}{l}\vec{n} \\
\stackrel{n}{+} \\
\stackrel{+}{\tilde{H}} \\
i \\
i\end{array}$ & 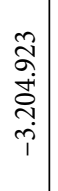 & \begin{tabular}{c}
$\vec{b}$ \\
\multirow{+}{*}{} \\
$\dot{n}$ \\
$\tilde{O}$ \\
$\dot{+}$
\end{tabular} & 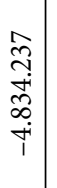 & $\begin{array}{l}\vec{\infty} \\
- \\
\vec{e} \\
\hat{0} \\
\dot{0} \\
1\end{array}$ \\
\hline 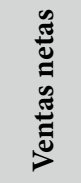 & 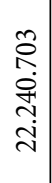 & 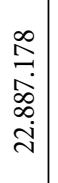 & $\begin{array}{l}\overrightarrow{0} \\
0 \\
\dot{0} \\
\dot{\vec{n}} \\
\infty \\
\ddot{\sim}\end{array}$ & $\begin{array}{l}\hat{m} \\
\cdots \\
\infty \\
\hat{n} \\
n \\
\stackrel{\sim}{\sim}\end{array}$ & \begin{tabular}{l|}
$\stackrel{n}{n}$ \\
$\tilde{n}$ \\
$\infty$ \\
$\infty$ \\
$\tilde{n}$ \\
$\stackrel{n}{n}$
\end{tabular} & 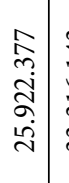 & 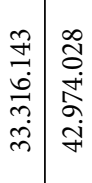 & 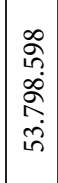 & 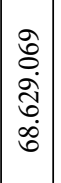 & 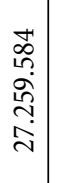 & 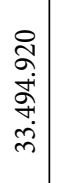 & 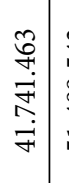 & 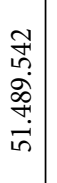 & 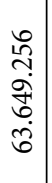 \\
\hline 夛 & $\vec{\overbrace{}}$ & $\stackrel{\infty}{\stackrel{\sim}{\circ}}$ & 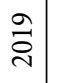 & ণ্ণి & $\overrightarrow{\widetilde{઼}}$ & $\stackrel{\curvearrowright}{\stackrel{\sim}{2}}$ & 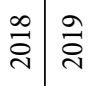 & ઠ્ડ & $\overrightarrow{\widetilde{d}}$ & $\stackrel{ }{\stackrel{ }{\nu}}$ & $\stackrel{\infty}{\overrightarrow{2}}$ & $\stackrel{\overbrace{}}{\vec{\sim}}$ & ્ֻণి & $\overrightarrow{\widetilde{d}}$ \\
\hline 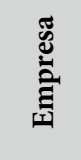 & & & $\begin{array}{l}\dot{s} \\
\dot{\Delta} \\
\dot{U} \\
\dot{U}\end{array}$ & & & & 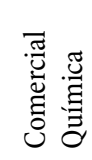 & & & & & 苞 & & \\
\hline
\end{tabular}


Para el cálculo del WACC de cada empresa, primero se obtuvo la beta, por lo cual se identificó el sector que más se asemeja a la actividad económica con respecto a las betas de los países emergentes y se aplicó la fórmula propuesta. En la Tabla 7 se muestran los resultados del ejercicio.

Tabla 7. Betas estimadas de las empresas de estudio

\begin{tabular}{l|l|c|c|c|c}
\hline \multicolumn{1}{c|}{ Empresa } & Sector & $\begin{array}{c}\text { Sector } \\
\text { comparable }\end{array}$ & $\begin{array}{c}\text { Beta } \\
\text { desapalancado } \\
\text { del sector }\end{array}$ & $\begin{array}{c}\text { Tasa de } \\
\text { impuesto } \\
\text { promedio }\end{array}$ & $K_{U}$ \\
\hline CJC S. A. S. & $\begin{array}{l}\text { Procesamiento y } \\
\text { conservación de } \\
\text { carne y productos } \\
\text { cárnicos }\end{array}$ & $\begin{array}{c}\text { Food } \\
\text { Processing }\end{array}$ & 0.96 & $48.19 \%$ & $10.7 \%$ \\
\hline $\begin{array}{l}\text { Comercial } \\
\text { Química }\end{array}$ & $\begin{array}{l}\text { Importación y co- } \\
\text { mercialización de } \\
\text { Ltda. } \\
\text { (químias primas }\end{array}$ & $\begin{array}{c}\text { Chemical } \\
\text { (Basic) }\end{array}$ & 0.71 & $38.28 \%$ & $9.5 \%$ \\
\hline $\begin{array}{l}\text { Distrimarcas } \\
\text { S. A. S. }\end{array}$ & $\begin{array}{l}\text { Comercio al por } \\
\text { mayor de produc- } \\
\text { tos alimenticios }\end{array}$ & $\begin{array}{c}\text { Food } \\
\text { Wholesalers }\end{array}$ & 0.57 & $35.85 \%$ & $8.8 \%$ \\
\hline
\end{tabular}

Fuente: elaboración propia a partir de los estados financieros de las empresas objeto de estudio.

Una vez calculado las betas, previamente se había determinado la tasa libre de riesgo y la prima por riesgo de mercado, se estimó el costo del patrimonio y el costo de la deuda; debido a la poca información disponible, se estimó el WACC con el valor total del patrimonio y del pasivo. En la Tabla 8 se relaciona el WACC para cada empresa de estudio.

$7 \quad$ Se utilizó la tasa de impuesto promedio por los cambios en la normatividad colombiana. Para desapalancar la beta del sector se utilizó la tasa del impuesto estimada por Damodaran (2018). 
Tabla 8. WACC calculado de las empresas de estudio

\begin{tabular}{|c|c|c|c|c|c|c|c|}
\hline Empresa & Año & $\%$ & $\% \mathrm{E}$ & \% D & $k_{d} \%$ & $\begin{array}{c}\text { Tasa de } \\
\text { impuesto }\end{array}$ & WACC \\
\hline \multirow{6}{*}{ CJC S. A. S. } & 2016 & & 22.6 & 77.4 & & & \\
\hline & 2017 & 39.1 & 24.1 & 75.9 & 2.5 & $48.2 \%$ & $12.23 \%$ \\
\hline & 2018 & 36.8 & 25.6 & 74.4 & 2.5 & $48.2 \%$ & $12.21 \%$ \\
\hline & 2019 & 34.9 & 27.0 & 73.0 & 2.5 & $48.2 \%$ & $12.19 \%$ \\
\hline & 2020 & 33.1 & 28.5 & 71.5 & 2.5 & $48.2 \%$ & $12.17 \%$ \\
\hline & 2021 & 31.5 & 30.0 & 70.0 & 2.5 & $48.2 \%$ & $12.16 \%$ \\
\hline \multirow{6}{*}{$\begin{array}{l}\text { Comercial Química } \\
\text { Ltda. }\end{array}$} & 2016 & & 23.2 & 76.8 & & & \\
\hline & 2017 & 7.8 & 23.8 & 76.2 & 10.0 & $38.3 \%$ & $7.86 \%$ \\
\hline & 2018 & 7.8 & 24.3 & 75.7 & 10.0 & $38.3 \%$ & $7.89 \%$ \\
\hline & 2019 & 7.9 & 24.9 & 75.1 & 10.0 & $38.3 \%$ & $7.91 \%$ \\
\hline & 2020 & 7.9 & 25.4 & 74.6 & 10.0 & $38.3 \%$ & $7.93 \%$ \\
\hline & 2021 & 8.0 & 26.0 & 74.0 & 10.0 & $38.3 \%$ & $7.95 \%$ \\
\hline \multirow{6}{*}{ Distrimarcas S. A. S. } & 2016 & & 51,5 & 48.5 & & & \\
\hline & 2017 & 14.3 & 53.2 & 46.8 & 2.9 & $35.8 \%$ & $9.55 \%$ \\
\hline & 2018 & 13.9 & 54.9 & 45.1 & 2.9 & $35.8 \%$ & $9.56 \%$ \\
\hline & 2019 & 13.6 & 56.6 & 43.4 & 2.9 & $35.8 \%$ & $9.57 \%$ \\
\hline & 2020 & 13.3 & 58.3 & 41.7 & 2.9 & $35.8 \%$ & $9.58 \%$ \\
\hline & 2021 & 13.0 & 60.0 & 40.0 & 2.9 & $35.8 \%$ & $9.60 \%$ \\
\hline
\end{tabular}

Fuente: elaboración propia.

Luego, se aplicó el modelo de valoración a cada una de las empresas como se muestra en la Tabla 9. El valor de la empresa es mayor al valor de los activos y del patrimonio para las empresas Comercial Química Ltda. y Distrimarcas S. A. S., debido a que los flujos de caja libre son consistentes con el crecimiento de la empresa y la información financiera disponible. 
Tabla 9. Resultados valoración de las empresas

\begin{tabular}{|c|c|c|c|c|c|}
\hline Empresa & $g_{n}$ & $\sum_{t=1}^{n} \frac{F C L_{t}}{(1+W A C C)^{t}}$ & $\frac{F C L_{n+1}}{W A C C-g_{n}}$ & $\frac{\frac{F C L_{n+1}}{W A C C-g_{n}}}{(1+W A C C)^{n}}$ & $\begin{array}{c}\text { Valor } \\
\text { de la } \\
\text { empresa }\end{array}$ \\
\hline CJC S. A. S. & $0.28 \%$ & 37.307 & 45.517 & 25.607 & 62.914 \\
\hline $\begin{array}{l}\text { Comercial } \\
\text { Química } \\
\text { Ltda. }\end{array}$ & $1.63 \%$ & 8.953 .638 & 76.282.008 & 52.042 .753 & 60.996 .391 \\
\hline $\begin{array}{l}\text { Distrimarcas } \\
\text { S. A. S. }\end{array}$ & $1.57 \%$ & 1.400 .590 & \begin{tabular}{|l|}
8.510 .462 \\
\end{tabular} & 5.382 .491 & 6.783 .080 \\
\hline
\end{tabular}

Fuente: elaboración propia.

Sin embargo, la valoración de la empresa CJC S. A. S. es menor a su valor total de activo, esto obedece a que la pyme tiene demasiados activos corrientes concentrados en las cuentas por cobrar y sus márgenes de ganancia y flujo de efectivo son inferiores comparado con el valor total de la venta neta y del activo; también se aprecia que en la empresa en los períodos de análisis el flujo de caja libre ha disminuido e incluso ha sido negativo. Lo anterior indica que la empresa está teniendo problemas en la gestión de sus recursos operacionales y está acumulando activos que no están generando utilidad.

\section{Conclusiones}

La valoración de empresas entendida como el proceso por el cual se cuantifican los hechos económicos, la capacidad de generar flujos de efectivo, el nivel de riesgos, la trayectoria y los factores externos, permite a los inversionistas actuales y prospectos identificar el precio de las compañías.

Para las empresas que no cuentan con un valor de mercado como es el caso de aquellas que no cotizan en bolsa, es cada vez más útil la valoración, debido a la necesidad por conocer su valor para establecer un precio de venta de la compañía, compararse con respecto a la competencia y justificar el precio de la emisión de instrumentos de patrimonio, entre otros propósitos. 
En la cuantificación del valor es fundamental tener en cuenta el contexto económico, social y cultural de cada país e incluso región de cada empresa, por lo que se debe combinar información interna y externa que pueda afectar la continuidad de la empresa.

Los métodos para la valoración dependen del objetivo, intención de continuidad, trayectoria, entorno económico y de la información disponible; para la realización de esta herramienta de toma de decisiones es fundamental contar con información financiera y contable suficiente para analizar los datos históricos y realizar las proyecciones pertinentes.

De acuerdo con la forma como se obtiene la información se definen grupos a partir de información interna. En el balance general se pueden utilizar los métodos de valor contable, el de libros ajustado y el sustancial; y por medio del estado de resultados se usan los métodos basados en el múltiplo PER, el del valor de los dividendos y el de múltiplos financieros.

Con respecto al good will, se trabaja con información interna, pero a su vez con los intangibles que generan valor a la empresa, de igual forma el método basado en descuentos de flujo se combina con la tasa de riesgo. Finalmente, la valoración por opciones reales, aunque es la más flexible y permite la inclusión de intangibles, no es la más adecuada para las pymes debido a que la información no es de fácil acceso.

Como las pymes en Colombia solo están obligadas a reportar sus estados financieros sin presentar revelaciones y al no pertenecer a la bolsa de valores, esto genera una gran incertidumbre en los inversionistas, por lo tanto, tiene una mayor estimación en el riesgo. Es importante medir este tipo de empresas teniendo en cuenta su aporte al PIB y por su capacidad de generación de empleo, ya es el tipo de empresas que más se conforman en el país.

Acorde a los antecedentes de valoración, el contexto económico y la información disponible, el método utilizado para la cuantificación es el basado en el flujo de caja libre, además de ser uno de los más completos, 
tiene un mayor rigor académico y amplia aceptación en las empresas; sin embargo, para la realización de los pronósticos se requiere conocer la empresa tanto interna como externamente y contar con un mayor detalle de la información, por lo que debido a que no se tenía acceso público a algunas partidas contables y financieras, algunos datos fueron estimados.

La aplicación del modelo de valoración propuesto indica que es apropiado para aplicar en las empresas pyme ya que los resultados son coherentes con la información financiera y con la situación de las empresas. No obstante, se deben considerar las particularidades de cada una de las empresas para ajustar el método y ser más precisos.

De las tres pymes analizadas que pertenecen al sector comercial e industrial dos comercializan alimentos y una de ellas químicos; su importancia se deriva de la trayectoria y nivel de ingresos facturados en este sector debido a que en conjunto superaron para el 2016 los 64000 millones de pesos. Con el cambio de estándar contable varios de los indicadores financieros disminuyen debido a que la información se ajustó a condiciones de mercado; sin embargo, permite que la valoración tenga una mayor precisión y sea más oportuna para la toma de decisiones.

El modelo de valoración es favorable para las empresas Comercial Química Ltda. y Distrimarcas S. A. S., ya que su valoración es mayor al valor de los activos, esto obedece a que los flujos de caja libre son consistentes con el crecimiento de la empresa y la información financiera disponible. Sin embargo, para la empresa CJC S. A. S. su valoración es menor al activo debido a que los flujos de caja libre presentan una disminución o son negativos, es necesario que la empresa realice una gestión de sus recursos, optimice la recuperación de cartera para poder hacer inversiones que le generen ganancias y mejore así su valoración.

La aplicación del modelo de valoración sugiere que sirve para aplicarlo en la mayoría de las empresas pymes considerando las particularidades de cada una. También los resultados de la valoración son consistentes con la información financiera y con la situación de la empresa. 
Esta propuesta de valoración mejora su efectividad al contar con la información financiera incluyendo las revelaciones y detalle de los movimientos contables, para poder proyectar los flujos de caja y utilizar la información histórica de manera más precisa. Los inconvenientes por obtener los datos necesarios de manera externa indican que los requerimientos por parte de las entidades de vigilancia y control deben ser más rigurosos y extensos para que los usuarios que no tienen acceso a información privilegiada como los posibles inversionistas, entidades financieras, organizaciones académicas, etc., puedan utilizarla para diferentes análisis como es el caso de la valoración.

\section{Referencias}

Asociación Nacional de Instituciones Financieras. (2017). Centro de estudios económicos. Recuperado de ANIF página oficial http://anif.co/sites/default/ files/ago14-17.pdf

Banco de la República. (s. f.). Tasas de colocación. Recuperado de http://www. banrep.gov.co/es/tasas-colocacion

Barrientos, S., y Bedoya, D. (2017). Valoración de una mediana empresa usando flujo de caja libre (FCL) y flujo de caja en riesgo (CFaR). Revista espacios, 113-127.

Black, F., y Scholes, M. (1973). The Pricing of Options and Corporate Liabilities. Journal of Political Economy, 81(3), 637-654.

Caicedo, L., y Angulo, F. (2017). Principios de valoración económica fundamentados en la teoría financiera: estudio en Pyme transportistas Maracaibo-Venezuela. Económicas CUC, 38(1), 14-164.

Confecamaras Red de Cámaras de Comercio. (2015). Informe de Coyuntura Empresarial en Colombia. Bogotá D. C., Colombia: Confecámaras.

Ley 905. (2004). Desarrollo de la micro, pequeña y mediana empresa en Colombia. Diario Oficial n. ${ }^{\circ} 45.628$ de la República de Colombia, Bogotá D. C., Colombia, 2 de agosto de 2004.

Correa, J., Jaramillo, F., Ramírez, L., y Castaño, C. (2008). ¿Es factible en las pymes la valoración y la creación de valor? Lúmina, 20-46.

Cox, J. C., Ross, S. A., y Rubinstein, M. (1979). Option pricing: A simplified approach. Journal of Financial Economics, 7(3), 229. 
Damodaran, A. (2002). Investment Valuation. New York: Wiley.

Damodaran, A. (8 de enero de 2018). Beta, Unlevered beta and other risk measures for Emerging Markets. Recuperado de http://www.stern.nyu.edu/ adamodar/ $\mathrm{pc} /$ datasets/betaemerg.xls

Damodaran, A. (8 de enero de 2018). Country Risk Premiums. Recuperado de http:// www.stern.nyu.edu/ adamodar/pc/archives/ctryprem16.xls

Escobar, G., Arias, L., y Portilla, L. (2009). Modelo para medir la situación financiera en empresas Pyme. Scientia et Technica, 273-278.

Fernández, P. (2004). Company valuation methods. The most common errors in valuations. Recuperado de IESE Business School. University Navarra: http:// www.iese.edu/research/pdfs/DI-0449-E.pdf

Fernández. (2008). Metodos de valoracion de empresas. Business, 1-49.

Gordon, M. J. (1962). The investment, financing and valuation of the corporation. Homewood, Ill: Jstor.

Grupo AVAL. (26 de diciembre de 2017). Portal Financiero. Recuperado de https:// www.grupoaval.com/wps/portal/grupo-aval/aval/portal-financiero/renta-fija/ tes/datos-historicos

Gutiérrez, M., Asmay, O., y Umañan, B. (2011). Valor de empresa mediante flujos de caja proyectados. El caso del hipódromo de Concepción - Chile. Horizontes Empresariales, 1(10), 53-61.

Jaramillo, F. (2010). Valoración de empresas. Bogotá D. C., Colombia: Ecoe Ediciones.

Londoño, A., y Sánchez, J. (2017). Aplicación del Costo de Capital en las pymes en Colombia: una revisión. Science of Human Action, 167-190.

Moscoso, J., y Botero, S. (2013). Métodos de valoración de nuevos emprendimientos. Semestre Económico, 237-264.

Narváez Liceras, A. (2009). Valoración de empresas: en busca del precio justo. Contabilidad y Negocios, 4(7), 23-30.

Narváez, A. (2008). Valoración de empresas por el método de los rendimientos futuros. Gestión en el Tercer Mileno, 1(11), 35-40.

Noussan, G. (2012). ¿Cuánto vale mi empresa? IEEM Revista de Negocios, 26-33.

Palepu Krishna, H. P. (2010). Business Analysis \& Valuation - Using Financial Statements. Cengage Learning Australia Pty Limited, 2-29. 
Parra, A. (2013). Valoración de empresas: métodos de valoración. Revista de la Facultad de Ciencias Económicas Administrativas y Contables, 2(1), 84-100.

Rojo, A. (2009). Importancia de la valoración de la empresa familiar: Los problemas de liquidez. Revista de Estudios Empresariales. Segunda época, 38-61.

Rosenberg, T. (2001). A Note on Valuation for Venture Capital. Recuperado de https://www.google.com.co/url? sa $=t \& r c t=j \& q=\& e s r c=s \& s o u r c e-$ $=$ eb $\& c d=1 \& c a d=r j a \& u a c t=8 \& v e d=0$ ahU KEwiNxKWq4NDNA hVK4CYKHUuAD-8QFggdMAA\&url=http\%3A\%2F\%2Fmaktabat mepi.org\%2Fdownload\%2F3145\%2F695\%2FValuation\%2520Ang. doc\&usg=AFQjCNG_Oee-dS7imQqrhVnPJDdiCpT72g\&sig2=qG

Superintendencia de Sociedades. (1 de mayo de 2017). Asuntos Económicos $y$ Contables. Recuperado de https://www.supersociedades.gov.co/ SiteCollectionDocuments/2017/EE-\%201000\%20mas\%20grandes\%20G1\%20 Y\%20G2_IDZ_10VI17\%20Final.pdf

Velásquez, F. (2004). La estrategia, la estructura y las formas de asociación: fuentes de ventaja competitiva para las pymes colombianas. Estudios gerenciales, 20(93), 73-97.

Vélez, I. (2002). Costo de capital para firmas no transadas en bolsa. Academia. Revista Latinoamericana de Administración, (29), 45-75.

Vélez-Pareja, I., y Tham, J. (2009). Market Value Calculation And The Solution Of Circularity Between Value And The Weighted Average Cost Of Capital Wacc. RAM. Revista de Administração Mackenzie, 6(10), 101-131.

Weston, F., y Copeland, T. (1994). Manual de Administración Financiera (Vol. I). Bogotá D. C., Colombia: McGraw-Hill. 